\title{
EXISTENCE AND NONEXISTENCE OF RADIAL LIMITS OF MINIMAL SURFACES
}

\author{
KIRK E. LANCASTER
}

(Communicated by Walter Littman)

\begin{abstract}
A bounded solution of the minimal surface equation is constructed which has no radial limits at a boundary point.
\end{abstract}

\section{INTRODUCTION}

The Dirichlet problem for the minimal surface equation consists of determining a function $f=f(x, y)$ satisfying the equation

$$
\left(1+f_{y}^{2}\right) f_{x x}-2 f_{x} f_{y} f_{x y}+\left(1+f_{x}^{2}\right) f_{y y}=0
$$

in a domain $\Omega$ and taking on assigned values on the boundary of $\Omega$. The boundary behavior of solutions of the Dirichlet problem has been well studied (e.g. $[5,6,7,8,9,13,15,16,19])$; when $\Omega$ is convex and the assigned boundary values are continuous, the (unique) solution is in $C^{0}(\bar{\Omega})$ [16], but this is generally false when $\Omega$ is not convex (e.g. the intriguing example of Simon [20]). If $\Omega$ is locally convex at each point of $\partial \Omega$ except one, say $N$, and the assigned boundary values are continuous, then the (generalized) solution of the Dirichlet problem will (probably) not be continuous at $N$ and yet will have radial limits at $N$ from each direction in $\Omega[3,10,11]$. If the assigned boundary values have a jump discontinuity at $N$, the (generalized) solution continues to have radial limits $[11,12]$.

Suppose $f$ is a bounded solution of the minimal surface equation in a domain $\Omega, N \in \partial \Omega$, and " $\left.f\right|_{\partial \Omega}$ " is not defined at $N$ (e.g. [20]). How does $f$ behave "at $N$ "? In this note we will construct a bounded solution of the minimal surface equation which has no radial limits at a point of the boundary. We also have some comments on the existence of radial limits.

Received by the editors November 15, 1988.

1980 Mathematics Subject Classification (1985 Revision). Primary 35J65, 35J67; Secondary $53 \mathrm{~A} 10$.

Key words and phrases. Nonparametric minimal surface, radial limit.

The author was supported, in part, by a 1987 Wichita State University Summer Faculty Research Grant. 


\section{NONEXISTENCE OF RADIAL LIMITS}

Let $\Omega$ be a convex domain in the plane. Suppose $N=(0,0) \in \partial \Omega$ and, say, $\{(x, 0): 0<x<1\} \subseteq \Omega$. We will use a "gliding hump" argument to construct a bounded solution $f$ of the minimal surface equation in $\Omega$ such that $\lim _{x \rightarrow 0+} f(x, 0)$ does not exist.

First we need a lemma similar to a "localization" lemma for harmonic functions.

Lemma. Let $\varepsilon>0$ and $h \in C^{2}(\Omega)$ be a solution of the minimal surface equation with $|h| \leq 2$. Then for each $\delta \in(0,1)$, there exists $g \in C^{2}(\Omega) \cap C^{0}(\Omega \cup\{N\})$ such that $g$ is a solution of the minimal surface equation in $\Omega, g(0,0)=2$, $|g| \leq 2$, and $\sup \{|h(x, y)-g(x, y)|:(x, y) \in \Omega$, $\operatorname{dist}((x, y), \partial \Omega) \geq \delta\}<\varepsilon$.

Proof. Let $g(x, y, t)$ be the solution of the Dirichlet problem with boundary values $h^{*}$ on $\partial \Omega \backslash B(N, t)$ and 2 on $\partial \Omega \cap B(N, t)$, where $B(N, t)$ is the disc about $N$ of radius $t$ and $h^{*}$ is the trace of $h$ on $\partial \Omega$. If $0<s<t$, then $h(x, y) \leq g(x, y, s) \leq g(x, y, t)$ for $(x, y) \in \Omega(g(\cdot, s)$ and $g(\cdot, t)$ can be considered as lower Perron solutions, for example) and so $g(\cdot, t)$ converges uniformly on compacta in $\Omega$ to a solution $H$ of the minimal surface equation as $t$ decreases to zero (e.g. [9], 329). Now $h(x, y) \leq H(x, y) \leq g(x, y, t)$ for $(x, y) \in \Omega, t>0$ and $g^{*}(x, y, t)=h^{*}(x, y)$ a.e. on $\partial \Omega \backslash B(N, t)$ so $H^{*}=h^{*}$ a.e. on $\partial \Omega$. Then the general maximum principle [14] implies $H=h$ on $\Omega$ and so $g(\cdot, t)$ converges uniformly on compacta to $h$. For $t_{0}$ close enough to zero, $g=g\left(\cdot, t_{0}\right)$ satisfies the conclusions of the lemma. Q.E.D.

We are in a position to prove the following

Theorem. Let $\Omega \subseteq R^{2}$ be an open convex set, $P \in \partial \Omega$, and $Q \in \Omega$. There exists a bounded solution $f$ of the minimal surface equation in $\Omega$ such that $f(x, y)$ has no limit as $(x, y)$ approaches $P$ along the line segment $P Q$.

Proof. We may assume $P=N=(0,0)$ and $Q=(1,0)$, as before. Pick $f_{1} \in C^{2}(\Omega) \cap C^{0}(\bar{\Omega})$ such that $\left|f_{1}\right| \leq 2$ and $f_{1}(0,0)=-2$. Let $r_{1} \in(0,1)$ such that $f_{1}\left(r_{1}, 0\right)<-1$. Let $\varepsilon_{1}=-\left(1+f_{1}\left(r_{1}, 0\right)\right)$. From the Lemma, we see that there exists $f_{2} \in C^{2}(\Omega) \cap C^{0}(\Omega \cup\{N\})$ such that $\left|f_{2}\right| \leq 2, f_{2}(0,0)=2$, and $\sup \left\{\left|f_{2}(x, y)-f_{1}(x, y)\right| \mid(x, y) \in \Omega, \operatorname{dist}((x, y), \partial \Omega) \geq \delta_{1}\right\}<\varepsilon_{1}$, where $\delta_{1}=\operatorname{dist}\left(\left(r_{1}, 0\right), \partial \Omega\right)$. Then $f_{2}\left(r_{1}, 0\right)<-1$. Now pick $r_{2} \in\left(0, r_{1}\right)$ such that $r_{2}<\frac{1}{2}$ and $f_{2}\left(r_{2}, 0\right)>1$.

In general, let $\varepsilon_{n}=\min _{1 \leq k \leq n}\left|f_{n}\left(r_{k}, 0\right)-(-1)^{k}\right|$ and $\delta_{n}=\operatorname{dist}\left(\left(r_{n}, 0\right), \partial \Omega\right)$. Use the Lemma to find $f_{n+1} \in C^{2}(\Omega) \cap C^{0}(\Omega \cup\{N\})$ with $f_{n+1}(0,0)=2(-1)^{n+1}$, $\left|f_{n+1}\right| \leq 2$, and $\sup \left\{\left|f_{n+1}(x, y)-f_{n}(x, y)\right|:(x, y) \in \Omega, \operatorname{dist}((x, y), \partial \Omega) \geq\right.$ $\left.\delta_{n}\right\}<\varepsilon_{n}$. Then pick $r_{n+1} \in\left(0, r_{n}\right)$ with $r_{n+1}<1 /(n+1)$ such that $f_{n+1}\left(r_{n+1}, 0\right)$ $>1$ if $n+1$ is even and $f_{n+1}\left(r_{n+1}, 0\right)<-1$ if $n+1$ is odd. It follows from the definition of $\varepsilon_{n}$ that $(-1)^{k} f_{n+1}\left(r_{k}, 0\right)>1$ for all $1 \leq k \leq n+1$. The sequence $\left\{f_{n}\right\}$ is uniformly bounded and so there is a subsequence, still denoted $\left\{f_{n}\right\}$, 
and a solution $f$ of the minimal surface equation such that $f_{n}$ converges to $f$ uniformly on compacta in $\Omega$ ([9], p. 323). Notice that $r_{n} \rightarrow 0$ as $n \rightarrow \infty$, $f\left(r_{n}, 0\right) \geq 1$ if $n$ is even, $f\left(r_{n}, 0\right) \leq-1$ if $n$ is odd, and $|f| \leq 2$. Thus $f$ is bounded and $\lim _{x \rightarrow 0} f(x, 0)$ does not exist. Q.E.D.

We may choose $\Omega$ to be symmetric about the non-negative $x$-axis and construct $f$ so that $f(x,-y)=f(x, y)$ and $\lim _{x \rightarrow 0} f(x, 0)$ does not exist. It is easy to see then that $f$ has no radial limits (e.g. [11]). A similar construction gives corresponding results for equations of bounded mean curvature and for nonparametric minimal hypersurfaces. In particular, this disproves the following conjecture. Set

$$
R f(\theta)=\lim _{t \rightarrow 0+} f(t \cos (\theta), t \sin (\theta))
$$

when this limit exists. Conjecture [11]: Let $f$ be a bounded solution of the minimal surface equation in a domain $\Omega$. Then $R f(\theta)$ exists for all $\theta$ for which $\{(r \cos (\theta), r \sin (\theta)): 0<r<\varepsilon\} \subset \Omega$, for some $\varepsilon>0$.

\section{REMARKS ON THE EXISTENCE OF RADIAL LIMITS}

Suppose $U$ is a connected, simply connected, bounded, open subset of $\mathfrak{R}^{2}$ with locally Lipschitz boundary such that $N=(0,0) \in \partial U$ and

$$
U=\{(r \cos (\theta), r \sin (\theta)): 0<r<r(\theta), \alpha<\theta<\beta\}
$$

for some $\alpha<\beta$. Let $U^{+}=\left\{(r \cos (\theta), r \sin (\theta)): \frac{1}{2}(\alpha+\beta)<\theta<\beta, 0<\right.$ $r<r(\theta)\}$ and set $\partial^{+} U=\partial U \cap \partial U^{+}$and $\partial^{-} U=\partial U \backslash \partial^{+} U$. Suppose $f \in$ $C^{0}(\bar{U} \backslash\{N\})$ is a bounded solution of the minimal surface equation. The graph of $f$ over $U, S$, then has finite area. Let us define

$$
a^{ \pm}=\liminf _{(x, y) \rightarrow(0,0)}\left\{f(x, y):(x, y) \in \partial^{ \pm} U\right\}
$$

and

$$
b^{ \pm}=\limsup _{(x, y) \rightarrow(0,0)}\left\{f(x, y):(x, y) \in \partial^{ \pm} U\right\} .
$$

Using a parametric representation of the graph of $f$ similar to that in [10] and [12], we can prove the following.

Proposition. Suppose $f \in C^{0}(\bar{U} \backslash\{N\})$ is a bounded solution of the minimal surface equation in $U$ and

$$
a=\max \left\{a^{-}, a^{+}\right\}>b=\min \left\{b^{-}, b^{+}\right\} .
$$

Then, for some $\alpha_{0}, \beta_{0} \in[\alpha, \beta]$ with $\alpha_{0}<\beta_{0}, R f(\theta)$ is defined and continuous on $\left(\alpha_{0}, \beta_{0}\right)$. Further, either

(i) $a=a^{+}\left(a^{-}\right), R f(\theta)$ is increasing (decreasing) on $\left(\alpha_{0}, \beta_{0}\right)$ and

$$
\lim _{\theta \rightarrow \alpha_{0}+} R f(\theta)=b^{-}\left(a^{-}\right) \quad \text { and } \quad \lim _{\theta \rightarrow \beta_{0}-} R f(\theta)=a^{+}\left(b^{+}\right)
$$

or 
(ii) there exists $\gamma \in\left(\alpha_{0}, \beta_{0}-\pi\right)$ such that $R f(\theta)$ is increasing (decreasing) on $\left(\alpha_{0}, \gamma\right]$, constant on $[\gamma, \gamma+\pi]$, and decreasing (increasing) on $\left[\gamma+\pi, \beta_{0}\right)$ and

$$
\lim _{\theta \rightarrow \alpha_{0}+} R f(\theta)=b^{-}\left(a^{-}\right) \quad \text { and } \quad \lim _{\theta \rightarrow \beta_{0}-} R f(\theta)=b^{+}\left(a^{+}\right) \text {. }
$$

In particular, if $z \in(b, a)$, then for some $\theta_{z} \in\left(\alpha_{0}, \beta_{0}\right), R f\left(\theta_{z}\right)=z$.

A similar result can be obtained for nonparametric surfaces of (bounded) prescribed mean curvature (using a parametrization similar to that in [4]).

Proof. Since the area of $S$ is finite, the Dirichlet integral of $X, D(X)$, is finite. The proposition follows using Courant's lemma ([1], Lemma 3.1) and the "jump" in the boundary values of $f$ "at $N$ ". Q.E.D.

\section{Examples.}

1. Let $U=\left\{(x, y): x^{2}+y^{2}<1, y>0\right\}$ and let $f \in C^{2}(U) \cap C^{0}(\bar{U} \backslash\{N\})$ be the solution of the minimal surface equation in $U$ with $f(x, 0)=0$ for $x \in(0,1), f(x, 0)=9+\cos (2 \pi / x)$ for $x \in(-1,0)$, and $f(\cos (\theta), \sin (\theta))=$ $(10 / \pi) \theta$ for $0 \leq \theta \leq \pi$. Then $a^{-}=b^{-}=0, a^{+}=8, b^{+}=10, \alpha_{0}=0$, $\beta_{0} \geq 4 \pi / 5$, and conclusion (i) of the proposition holds. To see this, consider the function $g(r \cos (\theta), r \sin (\theta))=(10 / \pi) \theta$ whose graph is a helicoid. Since $f \leq g$ on $\partial U \backslash\{N\}, f \leq g$ in $U$. Since $\operatorname{Rg}(\theta)=(10 / \pi) \theta<8$ iff $\theta<4 \pi / 5$, we see that $R f(\theta)$ exists and $R f(\theta) \leq R g(\theta)$ if $0 \leq \theta<4 \pi / 5$. Thus $\beta_{0} \in[4 \pi / 5, \pi]$.

2. Let $U=\left\{(x, y): x^{2}+y^{2}<1, y>0\right.$ or $\left.x<0\right\}$. Then $\alpha=0$ and $\beta=3 \pi / 2$. Let $g \in C^{0}(\bar{U} \backslash\{N\})$ be a solution of the minimal surface equation in $U$ such that $g(x, 0)=9$ for $x \in(0,1), g(0, y)=-1$ for $y \in(-1,0)$, and $R g(\theta)=20$ (such a function $g$ can be obtained by making $g$ large enough on $\partial U \cap \partial B$, where $\left.B=\left\{(x, y): x^{2}+y^{2}<1\right\}\right)$. Let $f \in C^{0}(\bar{U} \backslash\{N\})$ be the solution in $U$ of the minimal surface equation such that $f=g$ on $\partial U \cap \partial B, f(x, 0)=10+\cos (\pi / x)$ for $x \in(0,1)$, and $f(0, y)=\cos (\pi / y)$ for $y \in(-1,0)$. Then $g \leq f$ in $U$. Now $a^{-}=9, b^{-}=11, a^{+}=-1$, and $b^{+}=1$. Since $\operatorname{Rg}(\pi)=20$, conclusion (ii) of the proposition holds; in fact, $R f(\theta)$ is increasing on $\left(\alpha_{0}, \gamma\right]$, constant $(\geq 20)$ on $[\gamma, \gamma+\pi]$, and decreasing on $\left[\gamma+\pi, \beta_{0}\right)$, for some $\gamma \in\left(\alpha_{0}, \beta_{0}-\pi\right) \subseteq(0, \pi / 2), \lim _{\theta \rightarrow \alpha_{0}+} R f(\theta)=11$, and $\lim _{\theta \rightarrow \beta_{0}-} R f(\theta)=1$. Since $R f(\theta) \geq R g(\theta)$ for $\theta \in\left(\alpha_{0}, \beta_{0}\right)$, we see that $0 \leq \alpha_{0} \leq \alpha_{1}$ and $\beta_{1} \leq \beta_{0} \leq 3 \pi / 2$, where $\alpha_{1} \in(0, \pi / 2)$ such that $\operatorname{Rg}\left(\alpha_{1}\right)=11$ and $\operatorname{Rg}\left(\beta_{1}\right)=1$.

In general, the example in section 1 shows that $R f(\theta)$ need not be defined for even one value of $\theta$. If we represent the graph of $f$ parametrically as in [10] (and [12]), then the z-coordinate function $z(w)(w=u+i v)$ need not have a radial limit at a boundary point, say 1 , of the parameter domain $D=\{w:|w|<1\}$ which $x(w)$ and $y(w)$ both map to 0 . How poorly behaved is $z(w)$ near 1 ? It can be shown that (a suitable restriction of) $z$ is in $h^{p}(D)$ for all $p<1, \tilde{z} \in C^{0}(\bar{D}), \tilde{z}_{\theta} \in h^{1}(D)$, and $z+i \tilde{z} \in H^{\infty}(D) \cap V M O$, where $\tilde{z}$ 
is the harmonic conjugate of $z$ (here we use the notation of [2] and [17]). Of course, if $z$ were a little nicer (e.g. $z \in h^{1}(D)$ or $\tilde{z}_{\theta} \in h^{p}(D)$ for some $p>1$ ), then $z$ would have a radial limit at 1 .

\section{ACKNOWLEDGMENTS}

The author wishes to acknowledge the contribution of David Ullrich, who suggested the "gliding hump" construction, and to thank Kenneth Hoffman and Donald Sarason for their interest.

\section{REFERENCES}

1. R. Courant, Dirichlet principle, conformal mapping, and minimal surfaces, Interscience, New York, 1950.

2. P. Duren, Theory of $H^{p}$ spaces, Academic Press, New York, 1970.

3. A. Elcrat and K. Lancaster, On the behavior of a nonparametric minimal surface in a nonconvex quadrilateral, Arch. Rational Mech. Anal. 94 (1986), 209-226.

4. B_ Boundary behavior of nonparametric surfaces of prescribed mean curvature near a reentrant corner, Trans. Amer. Math. Soc. 297 (1986), 645-650.

5. R. Finn, Remarks relevant to minimal surfaces and to surfaces of prescribed mean curvature, J. Analyse Math. 14 (1965), 139-160.

6. C. Gerhardt, Existence, regularity, and boundary behavior of generalized surfaces of prescribed mean curvature, Math. Z. 139 (1974), 173-198.

7. E. Giusti, Boundary behavior of nonparametric minimal surfaces, Indiana Univ. Math. J. 22 (1972), 435-444.

8. S. Hildebrandt, Boundary behavior of minimal surfaces, Arch. Rational Mech. Anal. 35 (1969), 47-82.

9. H. Jenkins and J. Serrin, Variational problems of minimal surface type II. Boundary value problems for the minimal surface equation, Arch. Rational Mech. Anal. 32 (1966), 321-342.

10. K. Lancaster, Boundary behavior of a nonparametric minimal surface in $R^{3}$ at a nonconvex point, Analysis 5 (1985), 61-69.

11. __ Boundary behavior of nonparametric minimal surfaces-some theorems and conjectures, 37-41, in Variational Methods for Free Surface Interfaces (P. Concus and R. Finn, editors), Springer-Verlag, New York, 1987.

12. __ Nonparametric minimal surfaces in $R^{3}$ whose boundaries have a jump discontinuity, Internat. J. Math. Sci. 11 (1988), 651-656.

13. J. C. C. Nitsche, On new results in the theory of minimal surfaces, Bull. Amer. Math. Soc. 71 (1965), 195-270.

14. __ Über ein verallgemeinertes Dirichletsches Problem für die Minimal flächengleichung und hebbare Unstetigkeiten ihrer Lösungen, Math. Ann. 158 (1965), 203-214.

15. __ The boundary behavior of minimal surfaces. Kellogg's theorem and branch points on the boundary, Invent. Math. 8 (1969), 313-333.

16. T. Radó, The problem of least area and the problem of Plateau, Math. Z. 32 (1930), 763-796.

17. W. Ramey and D. Ullrich, The pointwise Fatou theorem and its converse for positive pluriharmonic functions, Duke Math. J. 49 (1982), 655-675. 
18. D. Sarason, Toeplitz operators with piecewise quasicontinuous symbols, Indiana Univ. Math. J. 26 (1977), 817-838.

19. L. Simon, Boundary regularity for solutions of the nonparametric least area problem, Ann. of Math. 103 (1976), 429-455.

20. _ Boundary behavior of solutions of the nonparametric least area problem, Bull. Austral. Math. Soc. 26 (1982), 17-27.

Department of Mathematics and Statistics, Wichita State University, Wichita, KANSAS 67208 